\title{
DETERMINACIÓN DE TARIFAS ÓPTIMAS PARA GENERACIÓN DE ELECTRICIDAD AL SISTEMA INTERCONECTADO A PARTIR DE RESIDUOS DE BIOMASA, UTILIZANDO EL SOFTWARE RETSCREEN Y APLICATIVOS DEL PROYECTO BEFS RAPID APPRAISAL DE NACIONES UNIDAS PARA LA AGRICULTURA Y LA ALIMENTACIÓN
} (FAO)

\author{
DETERMINATION OF OPTIMAL RATES FOR ELECTRICITY \\ GENERATION IN- SYSTEM I TERCONECTADO FROM WASTE \\ BIOMASS, USING THE RETSCREEN SOFTWARE AND \\ APPLICATIONS OF RAPID APPRAISAL BEFS PROJECT UN \\ FOOD AND AGRICULTURE (FAO)
}

\begin{abstract}
Aldo Max Delgado Acevedo ${ }^{1}$, Henry García Bustamante ${ }^{2}$, Carlos Orbegozo Reto ${ }^{3}$, Roxana Orrego $\mathrm{Moya}^{4}$, María Aragón Palacios ${ }^{5}$
\end{abstract}

\begin{abstract}
RESUMEN
El Perú cuenta con una extensión de 82 ‘369, 713 hectáreas de aptitud forestal, 7’600,000 con capacidad para los cultivos agrícolas y un potencial de biomasa para la producción de energía estimado en 272 millones de toneladas métricas anuales [2]. Para fines energéticos, existen cuatro categorías: plantaciones bioenergéticas, residuos agrícolas, bostas de ganado y residuos sólidos urbanos. La tecnología de gasificación sería la más conveniente para los fines del estudio. Evaluando el potencial teórico de generación eléctrica con biomasa que se pueda interconectar a la red, se obtuvo como resultado dos fuentes: arroz (Cajamarca, Lambayeque y Piura) y caña de azúcar (Lambayeque, Piura y Lima). Con el fin de proyectar la demanda, se tomó como guía el Plan Referencial de Electricidad 2008 - 2017. Los supuestos considerados son los siguientes: Escenario de Crecimiento Medio: Tasa de crecimiento Anual 7.55\%; Escenario de Crecimiento Optimista: Tasa de crecimiento Anual 8.7\%; Escenario de Crecimiento Conservador: Tasa de crecimiento Anual 6.36\%. Para determinar el Potencial Energético por Regiones desagregado por proyectos, se utilizó el aplicativo FAO BEFS Rapid Apraisal y el software RETScreen. De los 17 proyectos analizados, las herramientas calcularon que solamente cuatro podrían ser parte de una cartera de proyectos viables: Agroindustria Paramonga, Agroindustria Caña Brava, Relleno Sanitario Huaycoloro y Gran Mercado Mayorista de Lima (Santa Anita). La principal barrera que se ha encontrado es que las tarifas tope que ha establecido el Gobierno en las últimas subastas de proyectos para generación con energías renovables, no llega siquiera a estar próxima a las tarifas optimas analizadas en este estudio.
\end{abstract}

Palabras clave.- Energía renovable, Biomasa, Bioenergía, Proyectos de biomasa.

\footnotetext{
${ }^{1}$ Ingeniero Químico, Docente Investigador en Energía de la Facultad de Ingeniería Química y Textil de la Universidad Nacional de Ingeniería, Lima-Perú ${ }^{2}$ Experto en Bioenergía, Ingeniero Químico de la Universidad Nacional de Ingeniería, Lima-Perú, Green Energy. ${ }^{3}$ Ingeniero Físico de la Universidad de Bucarest (Rumania). Green Energy. ${ }^{4}$ Experta en Bioenergía, Ingeniero Geográfica. Green Energy. ${ }^{5}$ Experta en Eficiencia energética, Ingeniero Químico. Green Energy.
} 


\begin{abstract}
Peru has a surface of 82'369,713 hectares of forestry suitability, 7'600,000 hectares with agriculture farming capacity and a biomass potential for energy generation estimated in 272 million annual metric tons [2]. For energy generation scope, there are four categories: bioenergy crops, agriculture waste, livestock waste and urban solid waste. Gasification technology would be more convenient for the scope of the study. Assessing the theoretical potential of energy generation with biomass for grid connection, the result was the identification of two sources: Rice (Cajamarca, Lambayeque and Piura) and sugar cane (Lambayeque, Piura and Lima). With the aim to design the energy demand, the Electricity Referential Plan 2008 - 2017 was used as base document. The considered assumptions were the following: Medium growth scenario: Annual growth rate 7.55\%; Optimist growth scenario: Annual growth rate 8.7\%; Conservative growth scenario: Annual growth rate 6.36\%. In order to determine the energy potential by regions disaggregated by projects, it was used the application FAO BEFS Rapid Appraisal and the software RETScreen. From the 17 analyzed projects, the tools calculated that only four could be part of a viable Project portfolio: Agroindustria Paramonga, Agroindustria Caña Brava, Huaycoloro Landfill and Gran Mercado Mayorista de Lima (Santa Anita). The main barrier found is that the top tariffs established by the Government in the last bids are even near to the optimum tariffs analyzed in this study.
\end{abstract}

Key words.- Renewable energy, Biomass, Bioenergy, Biomass projects.

\section{INTRODUCCIÓN}

El propósito del estudio llevado a cabo por encargo de la Dirección General de Eficiencia Energética (DGEE) del Ministerio de Energía y Minas (MINEM) fue determinar el potencial energético del recurso biomasa en las regiones de Arequipa, Cajamarca, Piura, Lambayeque, Junín y Lima, y formular una cartera de proyectos a nivel de pre factibilidad, usando como base la viabilidad energética de la biomasa en dichas regiones, especialmente para la generación de energía eléctrica.

Por su parte, el presente artículo muestra la metodología utilizada para determinar las tarifas óptimas que el gobierno central debería establecer para poder abastecer al sistema eléctrico interconectado (a través del mecanismo de subastas de generación con energías renovables no convencionales). Esta evaluación se realiza para una cartera de proyectos de generación eléctrica con biomasa identificados en las regiones de Lima, Arequipa, Piura, Lambayeque y Junín.

Con el fin de realizar esta evaluación, se han utilizado herramientas informáticas para evaluar tanto la cantidad de residuo de biomasa disponible para uso energético, así como las tecnologías de conversión de la biomasa en electricidad, tal como se explica a continuación.

\section{Metodología empleada para la determinación del portafolio de proyectos}

Para la evaluación de los proyectos de aprovechamiento en generación eléctrica de residuos de biomasa se empleó el siguiente procedimiento:

a) En el caso de los proyectos referidos a Ingenios Azucareros, Plantas Alcoholeras y Molinos de Arroz se utilizó la herramienta de FAO Agriculture Residues Tool [2] para la determinación de la Biomasa disponible para uso energético. La herramienta incorpora entre otros aspectos, factores de producción de residuos por unidad de producto para cultivos como la caña de azúcar, arroz, café, entre otros. Así mismo dispone de base de datos de Perú sobre producción de cultivos agrícolas, factores de conservación de residuos en el suelo para evitar la degradación de los suelos, entre otros. 


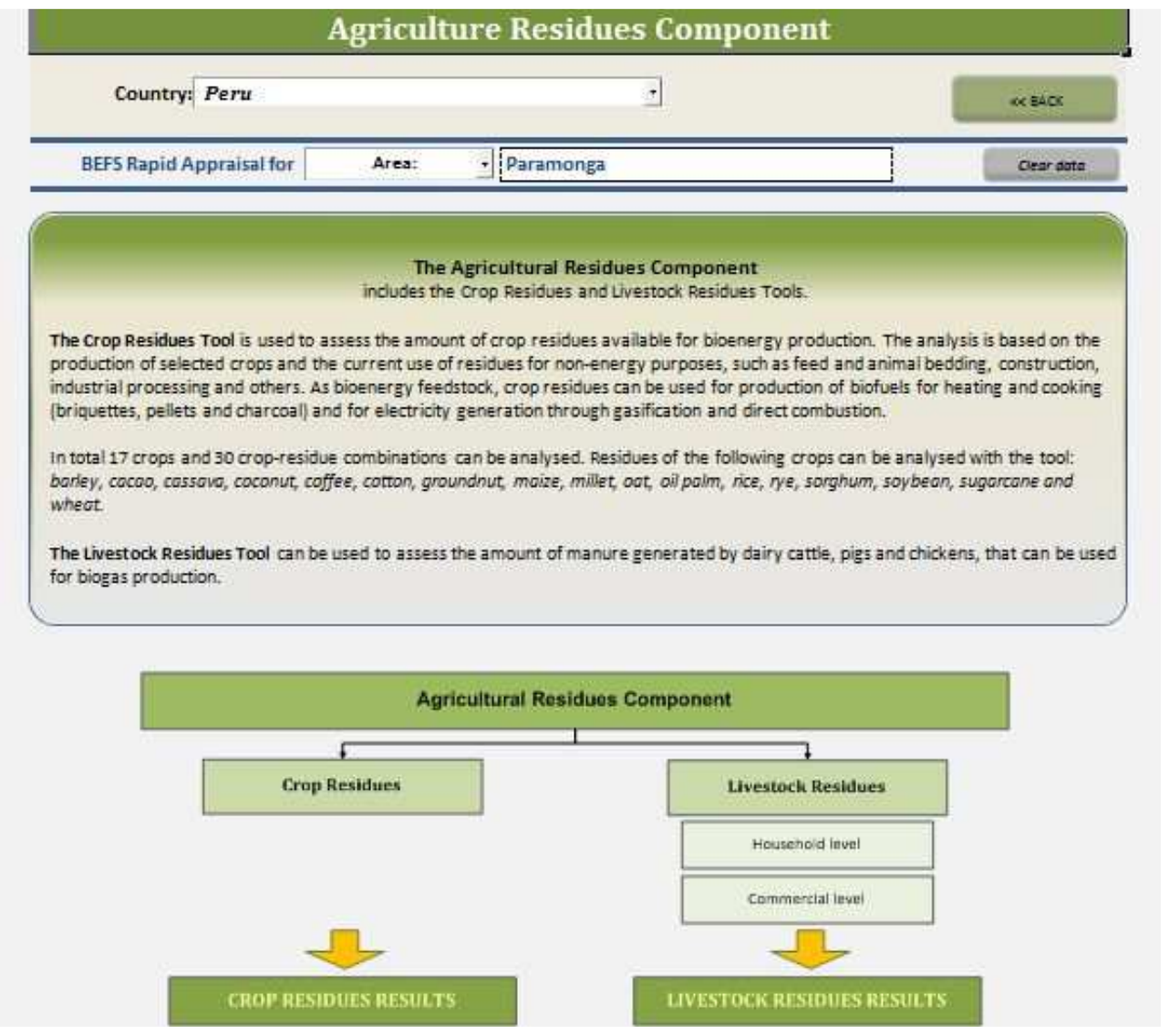

Fig. 1 Herramienta "Agriculture Residues Component" FAO - BEFS RA.

b) En el caso de residuos orgánicos provenientes de mercados de abastos, haciendas ganaderas, avícolas, camales y rellenos sanitarios, se ha determinado la cantidad disponible utilizando el software RETScreen [1] el cual en su pestaña Herramientas (Tools) tiene aplicaciones para determinación del biogás provenientes de este tipo de residuos considerando los datos meteorológicos de las locaciones evaluadas.

RETScreen Toois - Energy etficiency measures project
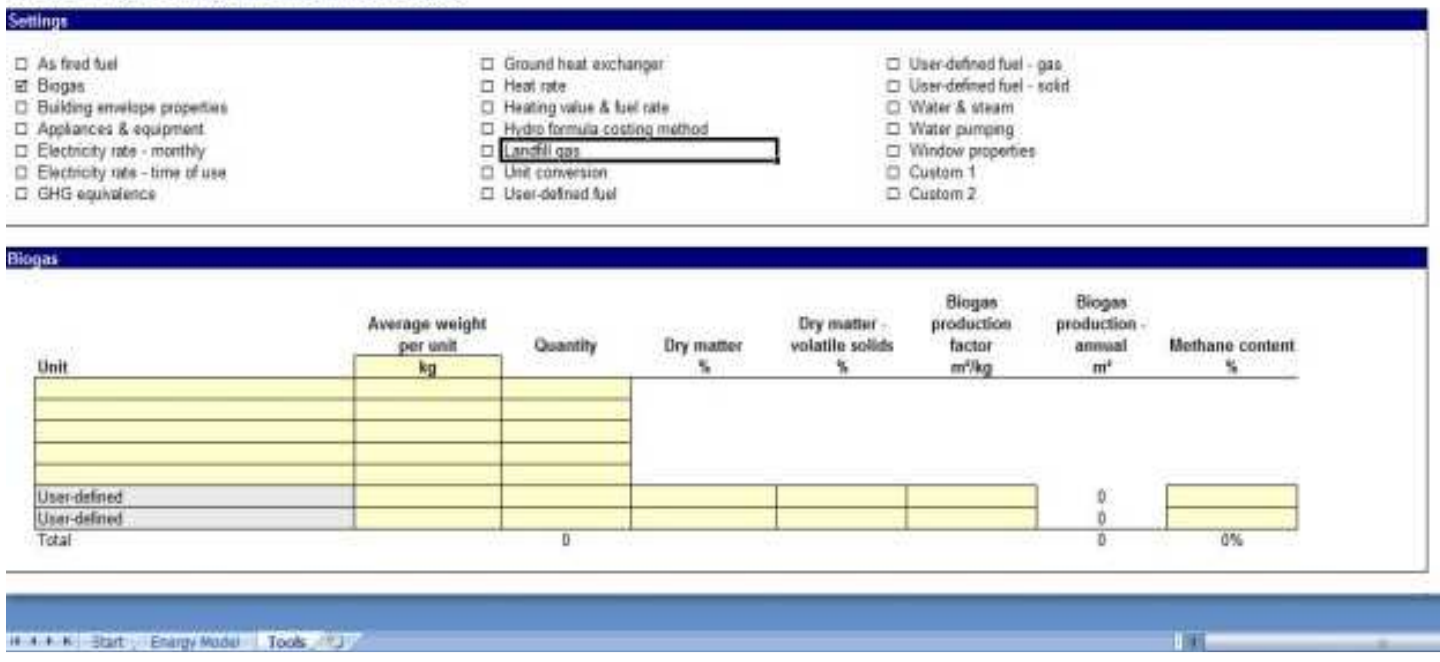

Fig. 2 Herramienta RETScreen (Aplicativo Biogás). 
c) Determinado la oferta de energía anual disponible a partir de los residuos ya sea a través de las herramientas de FAO BEFS Rapid Appraisal o de los aplicativos incluidos en el software RETScreen (Biogás o Landfill gas tool), lo siguiente es seleccionar la tecnología que se va a utilizar para el aprovechamiento energético mediante el software RETScreen. En el caso de los ingenios azucareros y plantas alcoholeras tomar en cuenta que se evaluaron dos casos. El primer caso referido al aprovechamiento de todo el bagazo disponible y el segundo caso referido al aprovechamiento del bagazo, follaje y topes. En este punto se evaluó las siguientes tecnologías, Tabla 1:

Tabla 1. Tecnologías consideradas.

\begin{tabular}{|c|c|c|}
\hline \multirow{2}{*}{ Tipo de Establecimiento } & \multicolumn{2}{|c|}{ Tecnología } \\
\hline & Suplir Demanda Térmica & Suplir Demanda Eléctrica \\
\hline Haciendas Ganaderas & $\begin{array}{l}\text { Calderas de Vapor operando con } \\
\text { Biogas }\end{array}$ & $\begin{array}{l}\text { Motor Reciprocante acoplado } \\
\text { con Biodigestor }\end{array}$ \\
\hline Granjas Avicolas & Hornos operando con biogas & $\begin{array}{l}\text { Motor Reciprocante acoplado } \\
\text { con Biodigestor }\end{array}$ \\
\hline Mercados mayoristas & $\begin{array}{l}\text { Calderas de Vapor operando con } \\
\text { Biogas }\end{array}$ & $\begin{array}{l}\text { Motor Reciprocante acoplado } \\
\text { con Biodigestor }\end{array}$ \\
\hline Camales & $\begin{array}{l}\text { Calderas de Vapor operando con } \\
\text { Biogas }\end{array}$ & $\begin{array}{l}\text { Motor Reciprocante acoplado } \\
\text { con Biodigestor }\end{array}$ \\
\hline Molinos de Arroz & $\begin{array}{l}\text { Calderas de Vapor operando con } \\
\text { cascarilla de arroz }\end{array}$ & $\begin{array}{l}\text { Turbina de Vapor acoplada con } \\
\text { la caldera }\end{array}$ \\
\hline $\begin{array}{l}\text { Agroindustria que procesa } \\
\text { esparragos }\end{array}$ & $\begin{array}{l}\text { Calderas de Vapor operando con } \\
\text { Biogas }\end{array}$ & $\begin{array}{l}\text { Motor Reciprocante acoplado } \\
\text { con Biodigestor }\end{array}$ \\
\hline $\begin{array}{l}\text { Ingenios Azucareros (Caso 1: } \\
\text { Aprovechamiento de Bagazo) }\end{array}$ & $\begin{array}{l}\text { Calderas de Vapor Bagaceras } \\
\text { convencionales operando con } \\
\text { bagazo }\end{array}$ & $\begin{array}{l}\text { Turbina de Vapor acoplada con } \\
\text { la caldera }\end{array}$ \\
\hline $\begin{array}{l}\text { Ingenios Azucareros (Caso 2: } \\
\text { Aprovechamiento de Bagazo, } \\
\text { Follaje y Topes) }\end{array}$ & $\begin{array}{l}\text { Calderas Bagaceras lecho } \\
\text { fluidizado operando con bagazo }\end{array}$ & $\begin{array}{l}\text { Turbina de Vapor acoplada con } \\
\text { la caldera }\end{array}$ \\
\hline $\begin{array}{l}\text { Plantas Alcoholeras (Caso 1: } \\
\text { Aprovechamiento de Bagazo) }\end{array}$ & $\begin{array}{l}\text { Calderas de Vapor Bagaceras } \\
\text { convencionales operando con } \\
\text { bagazo }\end{array}$ & $\begin{array}{l}\text { Turbina de Vapor acoplada con } \\
\text { la caldera }\end{array}$ \\
\hline $\begin{array}{l}\text { Plantas Alcoholeras (Caso 2: } \\
\text { Aprovechamiento de Bagazo, } \\
\text { Follaje y Topes) }\end{array}$ & $\begin{array}{l}\text { Calderas Bagaceras de Lecho } \\
\text { Fluidizado operando con bagazo }\end{array}$ & $\begin{array}{l}\text { Turbina de Vapor acoplada con } \\
\text { la caldera }\end{array}$ \\
\hline
\end{tabular}

d) Una vez seleccionada la tecnología para abastecer la demanda, se utilizó la data recogida de las visitas de campo o recabada mediante encuestas o en último caso accesible vía Internet para determinar la demanda anual ya sea de energía térmica o energía eléctrica.

e) Ya que se había estimado la demanda de energía por tipo de energía, la tecnología de abastecimiento y el recurso biomásico disponible para uso energético, se determina a través del software RETScreen, qué tanto la demanda interna es abastecida y si existen excedentes a ser comercializados a la red de sistema interconectado.

f) Calculada la oferta de energía disponible, se realiza una evaluación técnico-económica, así como una evaluación financiera, de sensibilidad (seleccionadas las variables más importantes). Finalmente se realiza una evaluación de riesgo mediante el método Montecarlo.

Los resultados de las evaluaciones observarlo en la Tabla 2 . 
Tabla 2. Resultado de la evaluación del portafolio de proyectos.

\begin{tabular}{|c|c|c|c|c|c|c|c|c|c|}
\hline \multirow[b]{2}{*}{ Región } & \multirow[b]{2}{*}{ Institución } & \multirow[b]{2}{*}{ Actividad } & \multicolumn{2}{|c|}{$\begin{array}{l}\text { Potencial Anual de } \\
\text { Aprovechamiento de Biomasa }\end{array}$} & \multicolumn{2}{|c|}{ Electricidad Generada } & \multicolumn{3}{|c|}{ Indicadores Financieros } \\
\hline & & & Cantidad & Rubro & $\begin{array}{c}\text { Potencia } \\
\text { instalada } \\
\text { (kW) }\end{array}$ & $\begin{array}{c}\text { Energía } \\
\text { Eléctrica } \\
\text { Generada } \\
\text { (MWh) }\end{array}$ & TIR (\%) & VAN (US\$̦) & $\begin{array}{c}\text { Periodo de } \\
\text { Recupero } \\
\text { (años) }\end{array}$ \\
\hline Arequipa & Fundo America & Fundo Ganadero & 450 & cabezas de ganado & 47 & 370 & 1.80 & -78185 & 23.50 \\
\hline Arequipa & $\begin{array}{l}\text { Fundo Pampa } \\
\text { Baja }\end{array}$ & Fundo Ganadero & 2000 & cabezas de ganado & 165 & 1301 & 1.30 & -316176 & 13.60 \\
\hline Junin & $\begin{array}{l}\text { Agroindustria del } \\
\text { Mantaro }\end{array}$ & $\begin{array}{l}\text { Producción de } \\
\text { Conservas de } \\
\text { Esparragos }\end{array}$ & 172800 & $\begin{array}{l}\text { Ton de Esparrago } \\
\text { Procesado }\end{array}$ & 220 & 1734 & -3.90 & -393434 & 13.10 \\
\hline Lambayeque & $\begin{array}{l}\text { Molinos de Arroz } \\
\text { (corredor Chiclayo } \\
\text { - Lambayeque) }\end{array}$ & $\begin{array}{l}\text { Molienda de } \\
\text { arroz }\end{array}$ & 176017 & $\begin{array}{l}\text { Ton de cascarilla } \\
\text { de arroz }\end{array}$ & 7354 & 57982 & 9.30 & 130136 & 6.80 \\
\hline Lambayeque & $\begin{array}{l}\text { Empresa Pomalca } \\
\text { (caso } 1 \text { ) }\end{array}$ & Ingenio Azucarero & 253776 & Bagazo disponible & 6255 & 54789 & 15.30 & 1773475 & 6.80 \\
\hline Lambayeque & $\begin{array}{l}\text { Empresa Pomalca } \\
\text { (caso 2) }\end{array}$ & Ingenio Azucarero & 421342 & $\begin{array}{l}\text { Bagazo, Follaje y } \\
\text { Topes disponibles }\end{array}$ & 10356 & 90717 & 10.70 & -1682867 & 11.80 \\
\hline Lambayeque & $\begin{array}{l}\text { Agroindustrial } \\
\text { Pucala (caso 1) }\end{array}$ & Ingenio Azucarero & 106320 & Bagazo disponible & 6953 & 60907 & 19.90 & 4176662 & 5.70 \\
\hline Lambayeque & $\begin{array}{l}\text { Agroindustrial } \\
\text { Pucala (caso 2) }\end{array}$ & Ingenio Azucarero & 176522 & $\begin{array}{l}\text { Bagazo, Follaje y } \\
\text { Topes disponibles }\end{array}$ & 10926 & 95711 & 3.00 & -11553533 & 11.00 \\
\hline Lima & Avinka & $\begin{array}{l}\text { Avicola - crianza } \\
\text { de aves }\end{array}$ & 1625000 & Producción de Aves & 800 & 7008 & 7.50 & -694386 & 11.20 \\
\hline Lima & Camal Conchucos & $\begin{array}{l}\text { Camal de } \\
\text { Animales }\end{array}$ & 1053 & $\begin{array}{l}\text { Ton Residuos } \\
\text { Organicos del } \\
\text { Camal }\end{array}$ & 150 & 1249 & 20.00 & 27980 & 10.00 \\
\hline Lima & Petramas & $\begin{array}{l}\text { Relleno Sanitario } \\
\text { de Huaycolloro }\end{array}$ & 1308832 & $\begin{array}{l}\text { Resdiuos Solidos } \\
\text { Urbanos } \\
\text { procesados }\end{array}$ & 5530 & 46624 & 20.90 & 1692946 & 4.60 \\
\hline Lima & $\begin{array}{l}\text { Mercado de Santa } \\
\text { Anita }\end{array}$ & $\begin{array}{l}\text { Mercado } \\
\text { Mayorista }\end{array}$ & 14600 & $\begin{array}{l}\text { Residuos solidos } \\
\text { organicos }\end{array}$ & 320 & 2804 & 26.40 & 2443870 & 4.10 \\
\hline Lima & $\begin{array}{l}\text { Agroindustrial } \\
\text { Paramonga (caso } \\
\text { 1) }\end{array}$ & Ingenio Azucarero & 253776 & Bagazo disponible & 140160 & 145124 & 23.60 & 22117312 & 3.60 \\
\hline Lima & $\begin{array}{l}\text { Agroindustrial } \\
\text { Paramonga (caso } \\
\text { 2) }\end{array}$ & Ingenio Azucarero & 421342 & $\begin{array}{l}\text { Bagazo, Follaje y } \\
\text { Topes disponibles }\end{array}$ & 28609 & 324664 & 10.20 & -12765655 & 6.00 \\
\hline Piura & Maple Ethanol & Planta Alcoholera & 1300000 & $\begin{array}{l}\text { Ton de caña } \\
\text { procesadas }\end{array}$ & 15735 & 153054 & 14.90 & 9015297 & 5.20 \\
\hline Piura & $\begin{array}{l}\text { Caña Brava (caso } \\
\text { 1) }\end{array}$ & Planta Alcoholera & 268171 & Bagazo disponible & 15735 & 153054 & 27.80 & 9928733 & 4.80 \\
\hline Piura & $\begin{array}{l}\text { Caña Brava (caso } \\
\text { 2) }\end{array}$ & Planta Alcoholera & 445243 & $\begin{array}{l}\text { Bagazo, Follaje y } \\
\text { Topes disponibles }\end{array}$ & 28609 & 314372 & 3.10 & -32025412 & 9.60 \\
\hline
\end{tabular}


Los proyectos sombreados en naranja representan a los que cumplen con los criterios de rentabilidad utilizados. Estos son los siguientes:

- Valor actual neto positivo

- Periodo de recupero no mayor a 5 años

- Tasa interna de retorno mayor al 9\% (Tasa de interés anual)

- Razón beneficio / costo mayor a 1 .
Tarifas mínimas para generación de electricidad con energías renovables requeridas para cubrir requisitos de rentabilidad a proyectos evaluados que no cumplen requisitos

Para aquellos proyectos que en la evaluación realizada no cubren los requisitos de rentabilidad establecidos, se analizó cual era la tarifa mínima requerida para poder cumplir estos requisitos, ver Tabla 3.

Tabla 3. Tarifas requeridas para proyectos evaluados.

\begin{tabular}{|c|c|c|c|c|c|}
\hline Región & Institución & $\begin{array}{l}\text { Tarifa Requerida para } \\
\text { cumplir requisitos de } \\
\text { rentabilidad } \\
\text { (Us\$/Mwh) }\end{array}$ & TIR (\%) & dores Financi & $\begin{array}{l}\text { ieros } \\
\text { Periodo de } \\
\text { Recupero } \\
\text { (años) }\end{array}$ \\
\hline Junin & Agroindustria del Mantaro & 203 & 25.10 & 343986 & 5.00 \\
\hline Lima & Avinka & 220 & 33.50 & 3361436 & 5.00 \\
\hline Lima & Camal Conchucos & 430 & 36.30 & 550636 & 5.00 \\
\hline Lima & Paramonga (caso 2) & 123 & 12.80 & 5532919 & 5.00 \\
\hline Lambayeque & $\begin{array}{l}\text { Molinos de Arroz (corredor } \\
\text { Chiclayo - Lambayeque) }\end{array}$ & 128 & 24.70 & 8072418 & 5.00 \\
\hline Piura & Caña Brava (caso 2) & 188 & 25.40 & 49154688 & 5.00 \\
\hline Piura & Maple Ethanol & 111 & 15.70 & 11353856 & 5.00 \\
\hline Lambayeque & Empresa Pomalca (caso 1) & 148 & 29.20 & 9037232 & 5.00 \\
\hline Lambayeque & Empresa Pomalca (caso 2) & 235 & 53.40 & 42398594 & 5.00 \\
\hline Lambayeque & Agroindustrial Pucala (caso 1) & 118 & 25.70 & 7186459 & 5.00 \\
\hline Lambayeque & Agroindustrial Pucala (caso 2) & 190 & 29.10 & 22242173 & 5.00 \\
\hline Arequipa & Fundo America & 330 & 31.50 & 154749 & 5.00 \\
\hline Arequipa & Fundo Pampa Baja & 270 & 58.40 & 1199172 & 5.00 \\
\hline
\end{tabular}

\section{CONCLUSIONES}

El principal potencial para generación de electricidad al sistema interconectado con biomasa alternativa, está centrado en los residuos del procesamiento de la caña de azúcar y del arroz. No obstante hay que considerar en el caso de la caña de azúcar, el aprovechamiento del follaje y topes junto con el bagazo en el caso de generación de electricidad demanda una tarifa mayor que el empleo de tecnologías de tipo más convencionales como el acople caldera bagacera tradicional - 
turbina de vapor. La razón son el uso de las tecnologías involucradas dado que se requiere de calderas de lecho fluidizado con un costo alrededor del doble de una caldera bagacera tradicional, además de los equipos de cosecha mecanizada, carguío y transporte de los campos a los ingenios.

En el caso de los residuos de arroz, la principal barrera que se tiene son los costos de acopio y transporte de la cascarilla a una planta de generación centralizada. No obstante, se ha encontrado una rentabilidad medianamente atractiva para una planta ubicada en el corredor Chiclayo-Lambayeque.

La principal barrera que se ha encontrado es que las tarifas tope que ha establecido el gobierno en las últimas subastas de proyectos para generación con energías renovables, no llega siquiera a estar próxima a las tarifas optimas analizadas en este estudio. Luego, se entiende que esto haya sido un motivo para la muy limitada cantidad de proyectos de generación con biomasa que han participado en las convocatorias.

El aplicativo FAO BEFS Rapid Appraisal y el software RETScreen, han sido de mucha ayuda para llegar a los resultados y a las conclusiones antes mencionadas. Ambas herramientas pueden ser utilizadas por investigadores, docentes universitarios, estudiantes y cualquier otro usuario experto con conocimientos previos sobre energía de la biomasa, para calcular y sustentar en forma científica el análisis de cualquier proyecto de biomasa. Ambas herramientas poseen una gran gama de aplicaciones en este campo, por lo que se recomienda su utilización a nivel académico.

\section{AGRADECIMIENTO}

El equipo consultor agradece a la Dirección General de Eficiencia Energética (DGEE) en la persona de su Directora, la Eco. Iris Cárdenas Pino, y a su equipo técnico, por las confianza depositada en nuestro trabajo y las facilidades proporcionadas para el cabal cumplimiento del estudio antes mencionado.

\section{REFERENCIAS}

1. Software RETScreen: //www.retscreen.net

2. Aplicativo FAO BEFS Rapid Appraisal: http://www.fao.org/energy/befs/86187/es/

3. García Bustamante, H., "Matriz Energética en el Perú y las Energías Renovables. VII. Barreras para el Desarrollo de la Bioenergía”. Primera Edición. Fundación Friedrich Ebert (FES) en colaboración con Derecho, Ambiente y Recursos Naturales (DAR). Lima-Perú, 2013.

4. Soluciones Prácticas ITDG. "Opción para la Producción y uso de Biodiesel en el Perú". Primera Edición. Lima-Perú, 2007.

5. BUN-CA. "Manuales sobre Energía Renovable: Biomasa". Primera edición. San José, Costa Rica, 2002.

6. Sánchez Albavera, F., Orrego Moya, R., "Tablero de Comando para la Promoción de Biocombustibles en el Perú". Naciones Unidas. Santiago de Chile 2007.

Correspondencia: corbegozo@energiaverde.pe

Recepción de documentos: Agosto 2014 Aceptación de documentos: octubre 2014 